IMA Journal of Applied Mathematics (2018) 00, 1-15

doi:10.1093/imamat/hxy007

\title{
Numerical computations of two-dimensional flexural-gravity solitary waves on water of arbitrary depth
}

\author{
TAO GAO \\ Department of Mathematical Sciences, University of Bath, Bath BA2 7AY, UK and \\ Department of Mathematics, University College London, London WCIE 6BT, UK \\ Jean-Marc Vanden-Broeck \\ Department of Mathematics, University College London, London WC1E 6BT, UK \\ AND \\ ZHAN WANG* \\ Institute of Mechanics, Chinese Academy of Sciences, Beijing 100190, China and \\ School of Engineering Sciences, University of Chinese Academy of Sciences, Beijing 100049, China \\ *Corresponding author: zwang@imech.ac.cn
}

[Received on 4 October 2017; revised on 27 January 2018; accepted on 28 February 2018]

\begin{abstract}
This work is concerned with flexural-gravity solitary waves on water of finite depth. The deformation of the elastic sheet is modelled based on the Cosserat theory of hyperelastic shells satisfying Kirchhoff's hypotheses. Both steady and unsteady waves are computed numerically for the full Euler equations by using a conformal mapping technique. Complete bifurcation diagrams of solitary waves are presented, and various dynamical experiments, including the evolution of unstable solitary waves and the generation of stable ones, are carried out via direct time-dependent simulations. In particular, we show that generalized solitary waves can also be excited by moving loads on the elastic cover.
\end{abstract}

Keywords: flexural-gravity; asymmetric waves; solitary waves.

\section{Introduction}

Waves propagating on water covered by an elastic sheet have recently received a significant research interest due to their wide applications in modelling floating ice (see Ashton (1986); Squire et al. (1996, 1988) and the references therein). Such waves are called flexural-gravity waves or hydroelastic waves. An in-depth understanding of this subject can enhance the engineering design of roads and aircraft runways in polar regions, as well as the construction of 'very large floating structures' in offshore and coastal areas. A main difficulty in studying theoretically these waves lies in the modelling of the deformation of the ice sheet (see Korobkin et al. (2011) for example) and a number of models have been proposed. The earliest model is linear water waves combined with the Euler-Bernoulli beam theory which is a good approximation for small-amplitude wave motions (see e.g. Squire et al. (1996)). Marko (2003) reported the observations of waves in ice events and his analysis showed that linear theories are not adequate for describing ice deflections of large amplitude. A hybrid formulation which combines the nonlinear water-wave theory and linear plate bending was developed by Părău \& Vanden-Broeck (2011) to model 3D hydroelastic waves of finite amplitude. On the other hand, in order to include the nonlinear 
effect due to elastic bending, a nonlinear model which is called the Kirchoff-Love (KL) model was widely used in the literatures since 1980s (Forbes (1986, 1988); Milewski et al. (2011); Părău \& Dias (2002); Vanden-Broeck \& Părău (2011)). More recently a novel nonlinear elastic model was proposed by Plotnikov \& Toland (2011), based on the Cosserat theory of hyperelastic shells satisfying Kirchhoff's hypotheses. In comparison to the KL model, the advantage of the Cosserat model is that it conserves elastic potential energy. Since this new model has been introduced, there has been a renewed interest in this problem and many investigations have already been performed by different authors, e.g. Gao \& Vanden-Broeck (2014), Gao et al. (2016), Guyenne \& Părău (2014) and Guyenne \& Părău (2012). In this paper, we restrict our attention to the Cosserat's model combined with the fully nonlinear water-wave theory in all the computations.

A number of experiments have been carried out at McMurdo Sound in Antarctica (Squire et al. (1988)) and at Lake Saroma in Japan (Takizawa, (1985, 1987, 1988)), which were summarized by Părău \& Dias (2002). As observed, the typical wavelength is $161 \mathrm{~m}$ in water with an average depth of $350 \mathrm{~m}$ at the former location (deep water case) while the corresponding value is $18.8 \mathrm{~m}$ with a depth of $6.8 \mathrm{~m}$ in Japan (shallow water case). Hence, to understand the influence of finite-depth effects in flexural-gravity wave motions is important for the locations geographically similar to Lake Saroma. There are two critical speeds in the flexural-gravity wave problem of finite depth. One is the so-called shallow water speed, $c_{0}=\sqrt{g h}$, where $g$ is the acceleration due to gravity and $h$ is the mean depth of water. The other one is the minimum $c_{\min }$ of the linear wave speed which is relevant to the nonlinear Schrödinger (NLS) equation and to the generation of wavepacket solitary waves. These two speeds differ considerably in deep water but are very close in the shallow-water case.

Nonlinear flexural-gravity waves on water of finite depth have been considered in the past by several groups. Gao \& Vanden-Broeck (2014) searched for periodic and generalized solitary waves by using a series truncation method. Guyenne \& Părău (2014) used a hodograph transformation to compute solitary waves and a high order spectral method to study the stability and dynamics of these solutions. Milewski \& Wang (2013) and Alam (2013) derived a Benney-Roskes-Davey-Stewartson type model for the 3D problem. Wang et al. (2014) computed lump solutions based on truncated models and pseudo-spectral methods. In this work, we solve the fully nonlinear Euler equations by using a time-dependent conformal mapping technique, which was pioneered by Dyachenko et al. (1996) and successfully used in deep water by Gao et al. (2016), Milewski et al. (2011, 2010) and Wang et al. (2014). Here we extend this work to the case of finite depth by following Choi \& Camassa (1999) and Li et al. (2004). Fully nonlinear steady and unsteady solutions are both considered in the present paper. A new finding on the excitation of generalized solitary wave is also presented at the end of the paper.

The rest of the paper is structured as follows. A detailed formulation is presented in Section 2 where we explain in detail the numerical technique. The numerical results are presented in Section 3. And finally a conclusion is given in Section 4.

\section{Formulation}

We consider a 2D irrotational flow of inviscid and incompressible fluid with density $\rho$ and depth $h$. The fluid body is bounded below by a solid horizontal boundary and covered above by an elastic sheet with flexural rigidity $D$. We choose a 2D Cartesian coordinate system, such that the positive $x$-direction is the wave propagation direction and the gravity is parallel to the $y$-axis pointing downwards. The deformation of the elastic sheet is denoted by $y=\zeta(x, t)$ which is unknown and varies with the time $t$. We introduce the velocity potential $\phi$ and streamfunction $\psi$. The governing equations can then be 

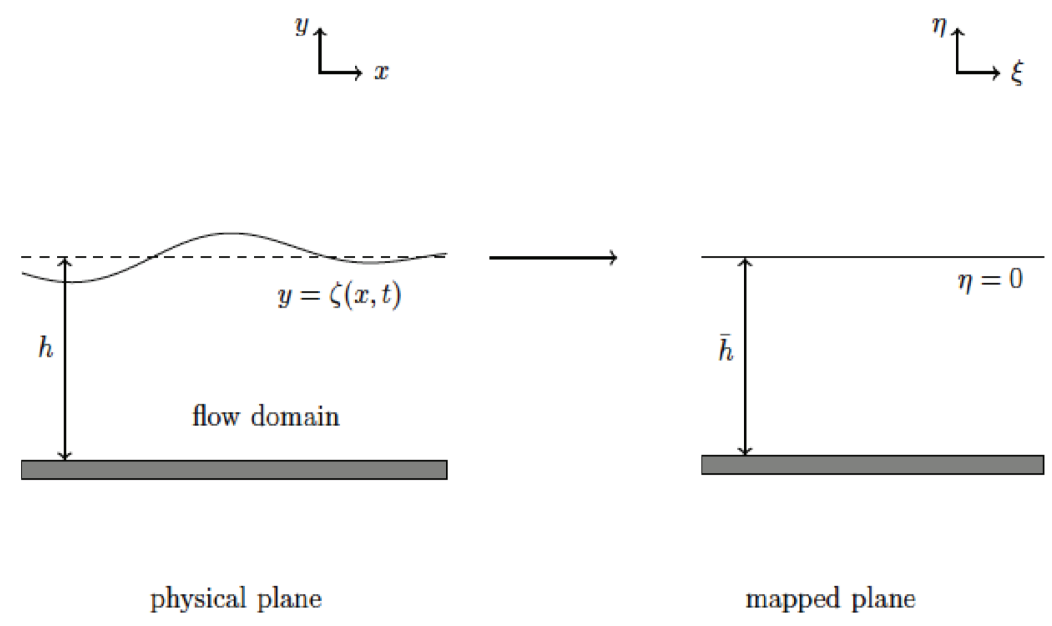

FIG. 1. Schematic of the conformal mapping.

expressed as follows

$$
\begin{aligned}
& \nabla^{2} \phi=0, \quad-h<y<\zeta(x, t), \\
& \zeta_{t}=\phi_{y}-\phi_{x} \zeta_{x}, \quad y=\zeta(x, t), \\
& \phi_{t}+\frac{1}{2}|\nabla \phi|^{2}+\zeta+\kappa_{s s}+\frac{1}{2} \kappa^{3}+\mathcal{P}_{e}=0, \\
& \phi_{y}=0, \quad y=-h,
\end{aligned}
$$

where we have chosen

$$
\left(\frac{D}{\rho g}\right)^{\frac{1}{4}},\left(\frac{D}{\rho g^{5}}\right)^{\frac{1}{8}}
$$

as the reference length and the reference time, respectively. Here $\mathcal{P}_{e}$ is the external forcing, $s$ is the arclength along the free surface and $\kappa$ denotes the curvature of the free surface. The total energy $\mathcal{E}$ of the system is given by

$$
\mathcal{E}=\frac{1}{2} \int_{\mathbb{R}} \int_{-h}^{\zeta}|\nabla \phi|^{2} \mathrm{~d} y \mathrm{~d} x+\frac{1}{2} \int_{\mathbb{R}} \zeta^{2} \mathrm{~d} x+\frac{1}{2} \int_{\mathbb{R}} \frac{\zeta_{x x}^{2}}{\left(1+\zeta_{x}^{2}\right)^{5 / 2}} \mathrm{~d} x .
$$

A time-dependent conformal mapping technique, pioneered by Dyachenko et al. (1996), is performed to transform the fluid domain onto a uniform strip of thickness $\bar{h}$ in the new $\xi-\eta$ plane, where the free surface is mapped to $\eta=0$. The schematic of the mapping is shown in Fig. 1. It is noted that solitary waves in our computations are approximated by long periodic waves with flat tails in the far field. We should emphasize that the wavelength $L$ is fixed in both planes, but the thickness of the strip 
in the conformal plane, $\bar{h}$, is varied in time to ensure conservation of mass. Following Choi \& Camassa (1999) and Li et al. (2004), the difference between $h$ and $\bar{h}$ is the mean value of the free surface elevation in the physical plane, i.e.

$$
h=\bar{h}(t)-\frac{1}{L} \int_{-L / 2}^{L / 2} \zeta(x, t) \mathrm{d} x .
$$

To ease the notations, the variables on the upper surface in the transformed plane are defined by

$$
\begin{aligned}
& X(\xi, t) \triangleq x(\xi, 0, t), \quad Y(\xi, t) \triangleq y(\xi, 0, t), \\
& \Phi(\xi, t) \triangleq \phi(\xi, 0, t), \quad \Psi(\xi, t) \triangleq \psi(\xi, 0, t) .
\end{aligned}
$$

After calculations similar to those presented in the studies by Choi \& Camassa (1999) and Li et al. (2004), the following equations are obtained:

$$
\begin{aligned}
& X_{\xi}=1-\mathcal{T}\left[Y_{\xi}\right], \\
& \Psi_{\xi}=-\mathcal{T}_{i}\left[\Phi_{\xi}\right], \\
& Y_{t}=Y_{\xi} \mathcal{T}\left[\frac{\Psi_{\xi}}{J}\right]-X_{\xi} \frac{\Psi_{\xi}}{J}, \\
& \Phi_{t}+\frac{\Phi_{\xi}^{2}-\Psi_{\xi}^{2}}{2 J}+Y+\frac{1}{2}\left[\frac{\kappa \xi \xi}{J}+\left(\frac{\kappa \xi}{J}\right)_{\xi}+\kappa^{3}\right]-\Phi_{\xi} \mathcal{T}\left[\frac{\Psi_{\xi}}{J}\right]+\mathcal{P}_{e}=0,
\end{aligned}
$$

where $J \triangleq X_{\xi}^{2}+Y_{\xi}^{2}$ is the Jacobian of the map and

$$
\begin{aligned}
\kappa= & \frac{X_{\xi} Y_{\xi \xi}-Y_{\xi} X_{\xi \xi}}{J^{3 / 2}}, \\
\kappa \xi= & \frac{Y_{\xi \xi \xi} X_{\xi}-X_{\xi \xi \xi} Y_{\xi}}{J^{3 / 2}}-\frac{3 \kappa\left(X_{\xi} X_{\xi \xi}+Y_{\xi} Y_{\xi \xi}\right)}{J} \\
\kappa \xi \xi= & \frac{X_{\xi \xi} Y_{\xi \xi \xi}+X_{\xi} Y_{\xi \xi \xi \xi}-Y_{\xi \xi} X_{\xi \xi \xi}-Y_{\xi} X_{\xi \xi \xi \xi}}{J^{3 / 2}} \\
& -\frac{6\left(X_{\xi} Y_{\xi \xi \xi}-Y_{\xi} X_{\xi \xi \xi}\right)\left(X_{\xi} X_{\xi \xi}+Y_{\xi} Y_{\xi \xi}\right)}{J^{5 / 2}} \\
& -\frac{3 \kappa\left(X_{\xi \xi}^{2}+Y_{\xi \xi}^{2}+X_{\xi} X_{\xi \xi \xi}+Y_{\xi} Y_{\xi \xi \xi}\right)}{J}+\frac{15 \kappa\left(X_{\xi} X_{\xi \xi}+Y_{\xi} Y_{\xi \xi}\right)^{2}}{J^{2}}
\end{aligned}
$$

The transformation $\mathcal{T}$ is defined by

$$
\mathcal{T}[f]=\frac{1}{2 \bar{h}} \int f\left(\xi^{\prime}\right) \operatorname{coth}\left(\frac{\pi}{2 \bar{h}}\left(\xi^{\prime}-\xi\right)\right) \mathrm{d} \xi^{\prime},
$$


with the Fourier symbol $i$ coth $(k \bar{h})$. The inverse $\mathcal{T}$-transform can be defined by

$$
\mathcal{T}_{i}[f]=-\frac{1}{2 \bar{h}} \int f\left(\xi^{\prime}\right) \operatorname{csch}\left(\frac{\pi}{2 \bar{h}}\left(\xi^{\prime}-\xi\right)\right) \mathrm{d} \xi^{\prime},
$$

with the Fourier symbol $-i \tanh (k \bar{h})$. In the limit of deep water $(\bar{h} \rightarrow \infty), \mathcal{T}$ becomes the Hilbert transform. For free waves travelling at a constant velocity $c$ (i.e. $\mathcal{P}_{e}=0$ ), all the functions depend on $x-c t$. Therefore, the evolution system can be reduced to

$$
\begin{aligned}
\Psi & =c Y, \\
\frac{c^{2}}{2}\left(\frac{1}{J}-1\right)+Y+\frac{1}{2}\left[\frac{\kappa \xi \xi}{J}+\left(\frac{\kappa \xi}{J}\right)_{\xi}+\kappa^{3}\right] & =0 .
\end{aligned}
$$

The total energy $\mathcal{E}$ for travelling waves can be written in terms involving only the surface variable $\xi$ in the transformed plane

$$
\mathcal{E}=\frac{1}{2} \int\left(\Phi_{\xi} \Psi+Y^{2} X_{\xi}+\frac{\left(Y_{\xi \xi} X_{\xi}-X_{\xi \xi} Y_{\xi}\right)^{2}}{J^{5 / 2}}\right) \mathrm{d} \xi
$$

As mentioned earlier we approximate solitary waves by long periodic waves so that the solutions can be written in the form of the Fourier series

$$
y=\sum_{n=-N}^{N}\left(a_{n}+i b_{n}\right) e^{i 2 \pi n \xi / L},
$$

with $b_{0}=0$. The value of $L$ is chosen sufficiently large so that the solution does not change within graphical accuracy if $L$ is further increased. The Fourier coefficients $a_{n}$ and $b_{n}$ are the unknowns. As we shall see in the next section there are two types of waves: symmetric and asymmetric waves. Symmetric waves are characterized by the existence of a vertical line in the physical plane which respect to which the profile is symmetric. Asymmetric waves are defined as waves for which no such vertical line exists. We describe here the numerical procedure for symmetric waves (the modifications for asymmetric waves will be described in the next section). We assume without loss of generality that the line of symmetry corresponds to $\xi=0$. It then follows that $a_{-n}=a_{n}$ and $b_{-n}=-b_{n}$ for $n \neq 0$. We use a spectral collocation method for spatial discretization. The $N$ collocation points are uniformly distributed along the $\xi$-axis, which provides discrete algebraic equations by projecting (2.20) onto each element of the basis $e^{i 2 \pi n \xi / L}$. All of the derivatives and the $\mathcal{T}$-transform are computed via Fourier multipliers while the nonlinear terms are computed in the real space. The energy $\mathcal{E}$ given in (2.21) is evaluated by the trapezoidal rule. The spatial step-size is chosen as $d \xi=0.05$ for steady computations. Typically we use $N=4096$ Fourier modes on a computational domain $[-100,100)$. The far field of the solitary waves is levelled at $y=0$. The wave height $H$ is defined by

$$
H=\left|\min _{x \in \mathbf{R}} \zeta(x)\right|
$$

where the value of $H$ is constrained by $H<h$. The algebraic equations are solved by Newton's method. Steady solutions in deep water, which were thoroughly investigated in the study by Gao et al. (2016), 
are chosen as the initial guess for solitary waves in finite depth. When $h=300$, only five iterations are usually required to achieve convergence with the residual error being less than $10^{-10}$. Based on a numerical continuation method using $h$ as a bifurcation parameter, we manage to find fully localized travelling-wave solutions in arbitrary depth.

Some preliminary insight into the problem can be gained by linearizing the dynamic boundary condition (2.20) by assuming that $Y, \Phi_{\xi}, \Psi_{\xi}$ are small and $J \sim 1, X_{\xi} \sim 1$. Solving the resultant linear equations yields the linear dispersion relation

$$
c^{2}=\left(\frac{1}{k}+k^{3}\right) \tanh (k h),
$$

(see e.g. Milewski \& Wang, 2013). It can be shown that $c$ always admits a global minimal value which we call the minimum of the phase speed $c_{\min }$. To ease the notation, we let $c_{h} \triangleq c_{\min }(h)$.

\section{Results}

\subsection{Steady solutions}

In this subsection, we aim to investigate free solitary waves, i.e. the external forcing $\mathcal{P}_{e}$ is switched off. We refer to the solitary waves as elevation waves when $\zeta(0)>0$ and as depression waves when $\zeta(0)<0$. The NLS equation for flexural-gravity waves in arbitrary depth, as shown below, was derived by Alam (2013) and Milewski \& Wang (2013) based on the Cosserat elastic model

$$
i A_{\tau}+\frac{\omega^{\prime \prime}}{2} A_{X X}+\mu|A|^{2} A=0
$$

where $\omega=k c$ is the angular frequency and

$$
\begin{aligned}
\mu= & \frac{5 \omega k^{6}}{2\left(1+k^{4}\right)}+\frac{\omega k^{2}}{4 \tanh ^{2} k h} \frac{\left(1+k^{4}\right) \tanh ^{4} k h-10\left(1+k^{4}\right) \tanh ^{2} k h+69 k^{4}+9}{15 k^{4}-\left(1+k^{4}\right) \tanh ^{2} k h} \\
& +\frac{\omega k^{2}}{2}\left(\frac{1+k^{4}}{\sinh ^{2} k h \cosh ^{2} k h}-4\right) .
\end{aligned}
$$

It is found that the coefficient $\mu$ of the nonlinear term changes sign at a critical value $h^{+} \simeq 233$, namely, the NLS equation is focussing, i.e. solitary waves can bifurcate from zero amplitude, when $h<h^{+}$ but defocussing, i.e. solitary waves can only bifurcate from finite amplitude, for $h>h^{+}$. We start with computing symmetric flexural-gravity solitary waves for $h=2$ and $h=300$. From the dispersion relation (2.24), the phase speed minimum can be easily found

$$
c_{2}=1.249944, \quad c_{300}=1.324676 .
$$

For $h=300$, the results are qualitatively similar to those found in deep water by Gao et al. (2016). Both branches of elevation waves and depression waves were found to exist at finite amplitudes (this is consistent with the fact that (3.1) is defocussing since $300>h^{+}$). It is shown in the right picture of Fig. 2 that the branch of depression waves is monotonic in the speed-amplitude diagram, while the branch of elevation waves features a snake-like bifurcation. For $h=2$ the detailed bifurcation diagrams are shown in the left of Fig. 2. The branches of solitary bifurcate from zero amplitude (this is again consistent with 

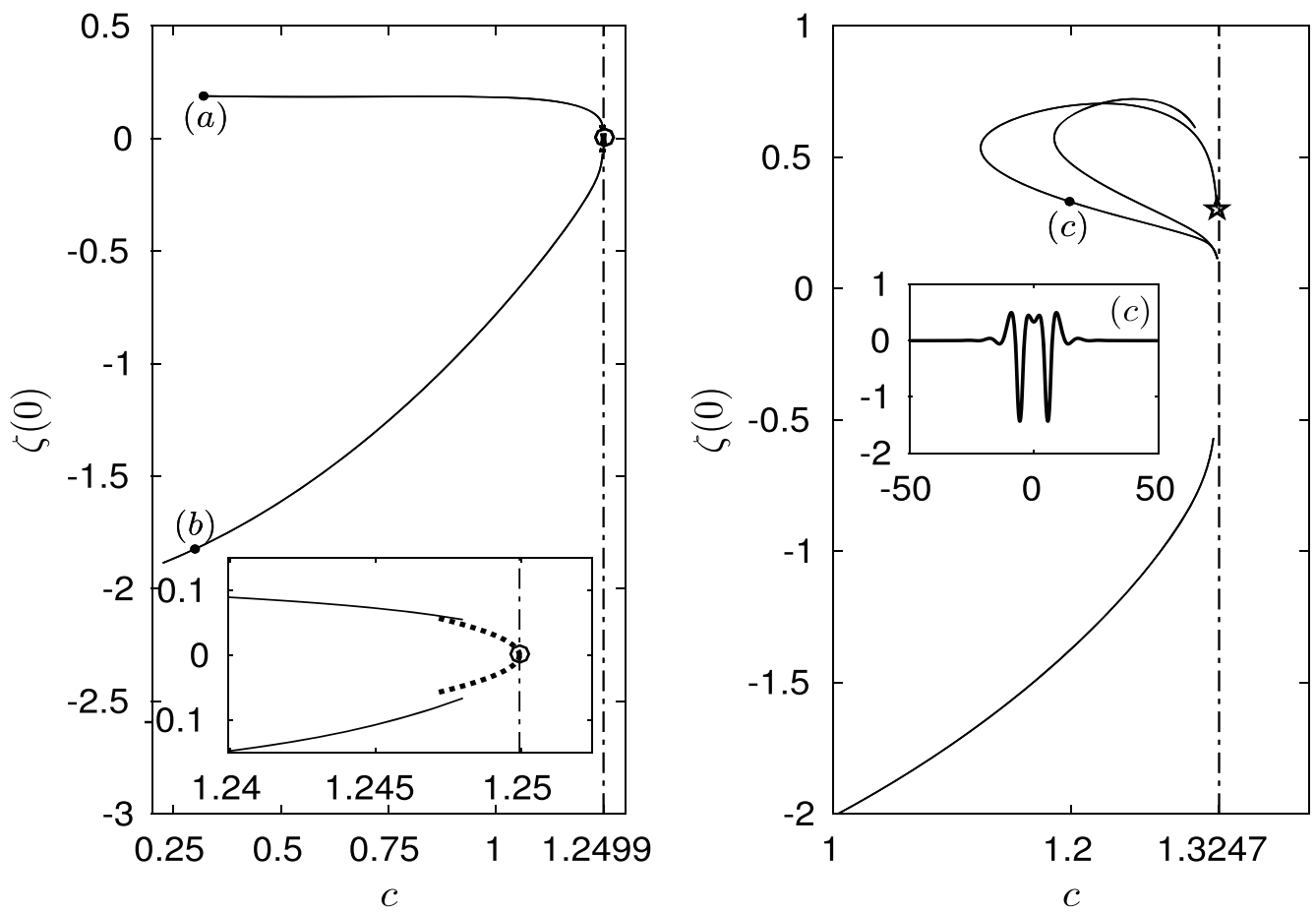

FIG. 2. Bifurcation diagrams of symmetric solitary waves for $h=2$ (left) and $h=300$ (right). The bifurcation speeds are sketched as vertical dash-dotted lines. The NLS prediction for $h=2$ is shown as the dotted line in the blow up graph. The bifurcation point of elevation solitary waves for $h=300$ is marked as a pentagram. Typical waves $(a)$ and $(b)$ for $h=2$, whose coordinates are $(0.32,0.189)$ and $(0.3,1.823)$, respectively, are presented in Fig. 3.
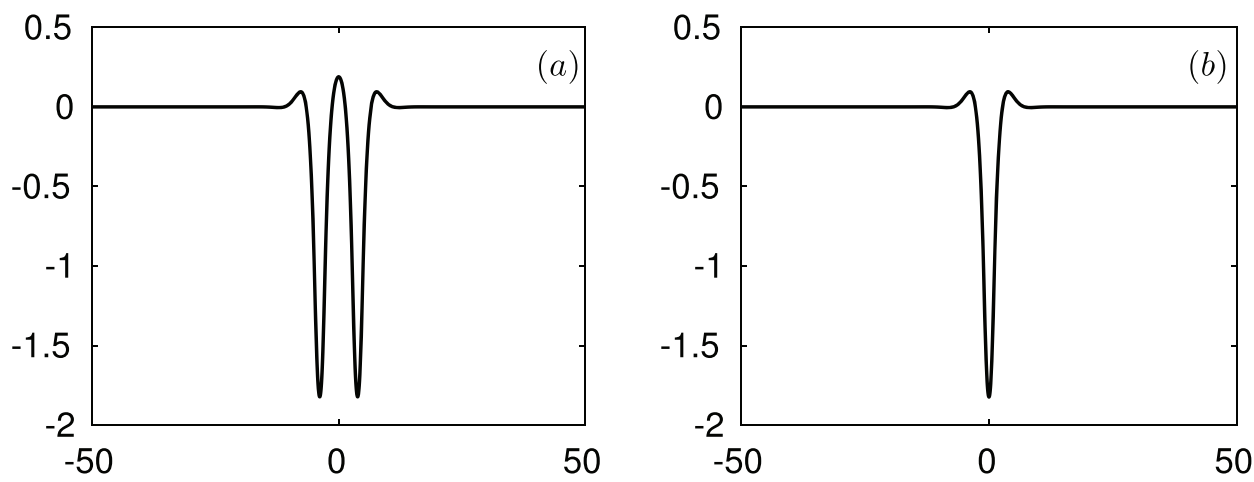

FIG. 3. Typical solitary wave profiles for $h=2$. The corresponding propagating speed is $c=0.32$ (left) and $c=0.3$ (right). The profiles are plotted in the physical plane.

the fact that (3.1) is focussing since $2<h^{+}$). The NLS prediction is a fairly good approximation near the bifurcation point (see the dotted line in the blow up graph). It is noted that the leading order of the weakly nonlinear theory underestimates the peak amplitude of depression solitary waves in the fully 

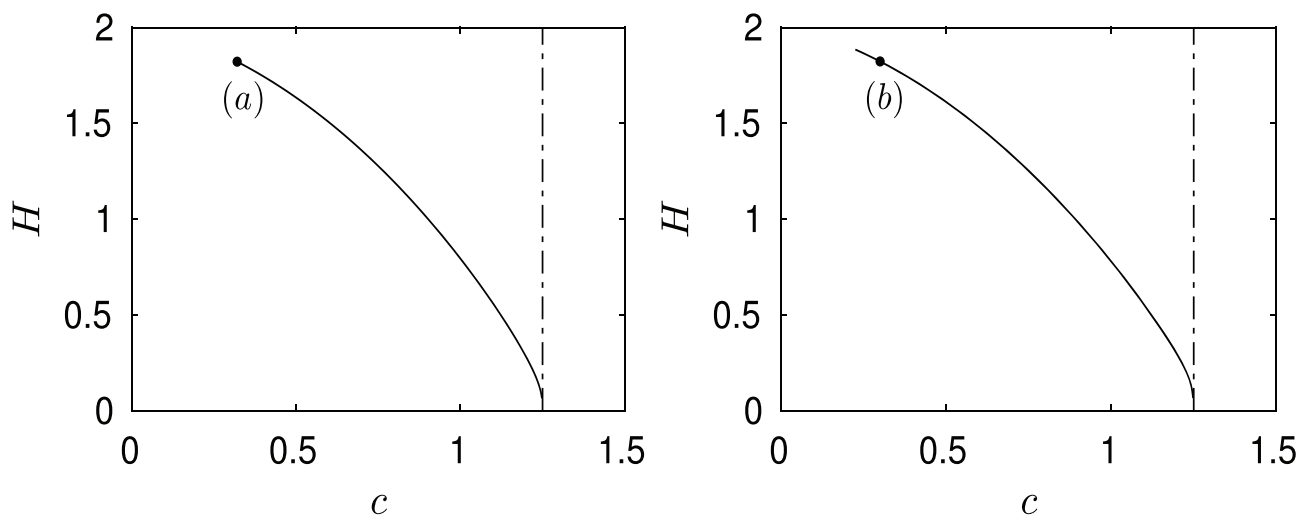

FIG. 4. $c-H$ diagrams of the elevation branch (left) and depression branch (right) for $h=2$. The bifurcation speeds are sketched as the vertical dash-dotted lines. The $(c, H)$ coordinates for $(a)$ and $(b)$ are $(0.32,1.713)$ and $(0.3,1.823)$, respectively.
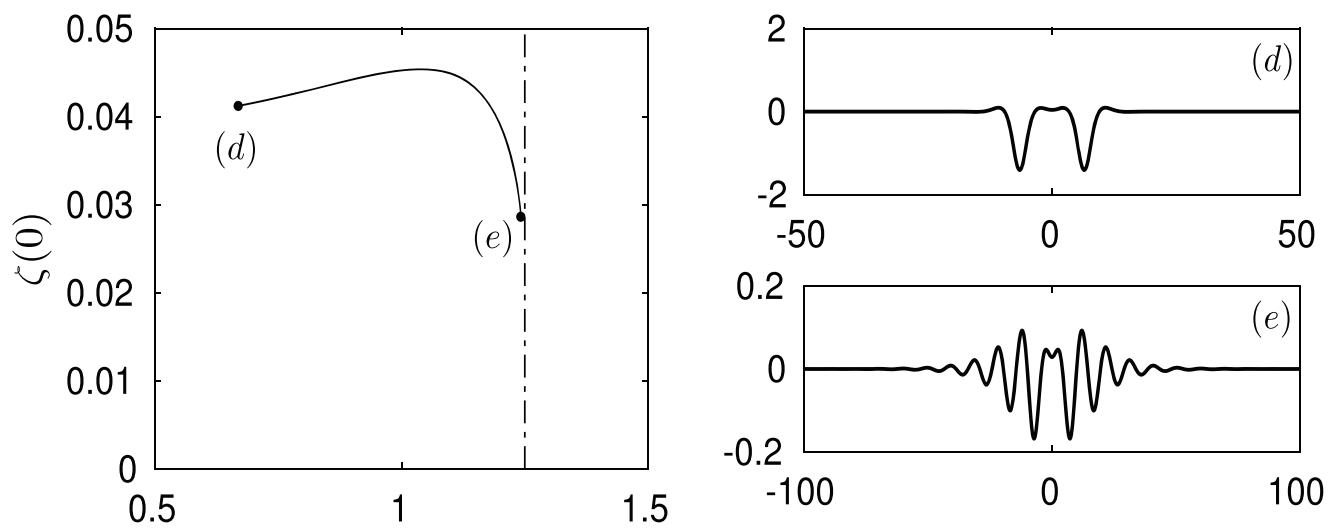

FIG. 5. Bifurcation diagram for elevation waves of the second kind. The coordinates of $(d)$ and $(e)$ are $(0.668,0.041)$ and $(1.242$, 0.029), respectively. The wave profiles plotted in the physical plane are presented on the right.

nonlinear computation. This discrepancy can be mitigated by introducing a second order correction as done in the study by Wang \& Milewski (2012) for the capillary-gravity wave problem. However, the branch of elevation waves, unlike the case of deep water, is a monotonic curve in the speed-amplitude diagram. Both depression and elevation waves approach a limiting configuration where the free surface touches the bottom. This is shown in the $c$ - $H$ diagram of Fig. 4, where we see that $H$ (defined by (2.23)) approaches 2 as one moves along the solution branches. The computations became harder to continue due to the touch-down singularity. An interesting question is do elevation waves, which feature two troughs connected by a small dimple (see solution $(c)$ in the right picture of Fig. 2), also exist for $h=2$ ? For easy reference, we denote this type of solutions as the elevation waves of the second kind. To answer this question, we started with the solution $(c)$ and chose $h$ as the parameter in the numerical continuation algorithm. It turns out that elevation waves of the second kind do exist for small $h$, and the complete solution branch is displayed in Fig. 5. We note that this curve does start from zero amplitude and is expected to turn near the point $(e)$ as it does as shown in Fig. 2 for $h=300$. In contrast to the deep-water case, the two elevation branches are separated for $h=2$. The breaking of the elevation branch takes place at the left end for a certain value of $h$ when the lowest troughs first touch the solid bottom. We 


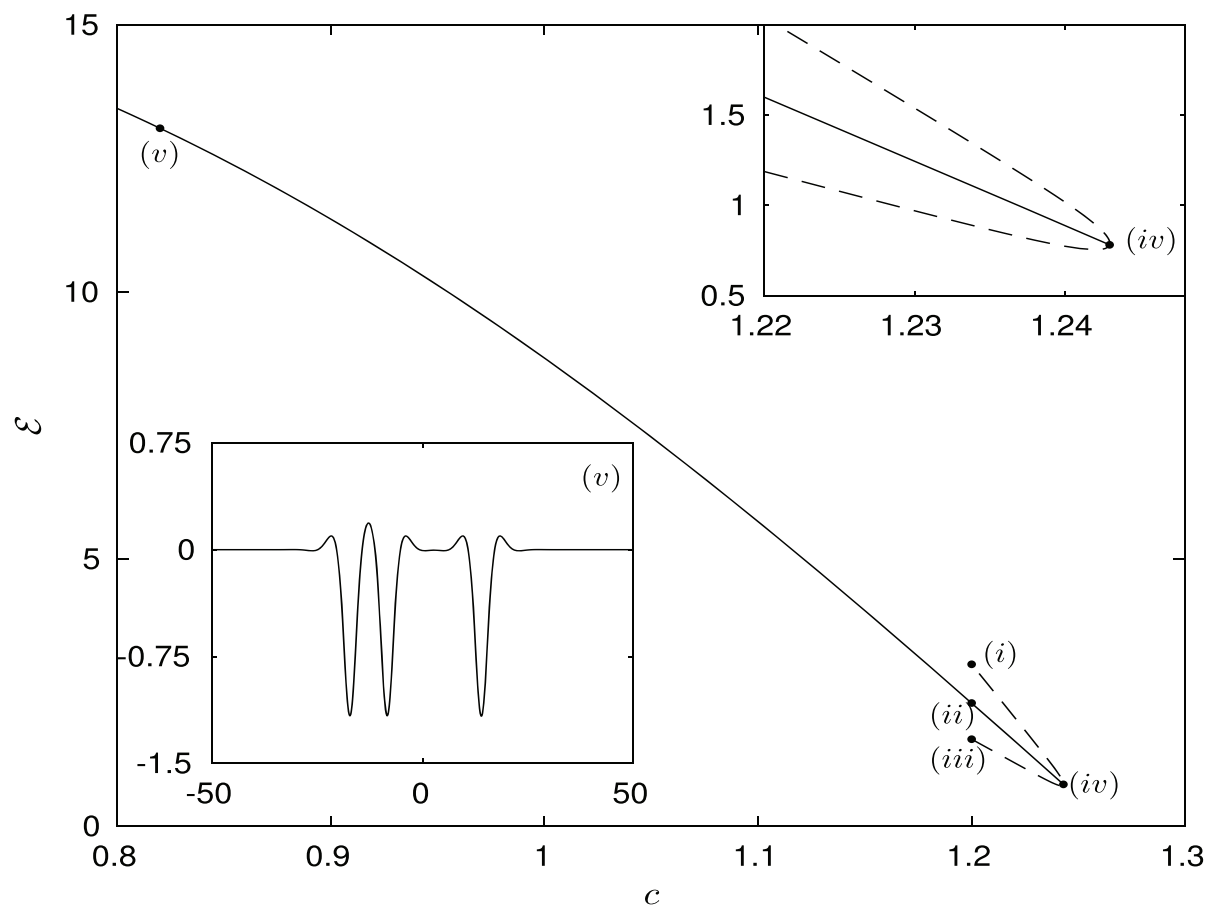

FIG. 6. Speed-energy bifurcation diagram of asymmetric solitary waves for $h=2$. A blow up graph near the bifurcation point is sketched on top left. The coordinates are (i) $(1.2,3.03),($ ii $)(1.2,2.304),($ iii $)(1.2,1.627),($ iv $)(1.243,0.7815)$ and $(v)(0.82,13.07)$.

encountered numerical difficulties in continuing the computations by further decreasing the translating speed from solution $(d)$. This is presumably because two troughs are far apart, and their separation is sensitive to perturbations. The stability of elevation waves on this branch will be studied in Section 3.2.

Next we attempt to compute asymmetric solitary waves by using the scheme introduced by Wang et al. (2014). Since the bifurcation structure for $h=300$ is similar to that in the case of deep water which has already been studied in the study by Gao et al. (2016), we restrict our focus in the case of $h=2$. The numerical procedure of Section 2 is modified by no longer assuming $a_{-n}=a_{n}$ and $b_{-n}=-b_{n}$ for $n \neq 0$. In other words all the values of $a_{n}$ and $b_{n}$ for $n$ positive and negative are now unknowns. It is shown in Fig. 6 that asymmetric solitary waves appear via a symmetry-breaking bifurcation near the phase speed minimum and that the speed-energy curve is monotonic for a small $h$. The asymmetric branch (solid curve) joins the symmetric branch (dashed curve) at the bifurcation point (iv) which is shown in detail in the blow up graph. The typical symmetric profiles of $(i-i v)$ are displayed in Fig. 7, where we observe multi-packet structures for both symmetric and asymmetric waves. The numerics became prohibitive for small $c$ due to the same reason as for the elevation branch of the second kind.

\subsection{Dynamics}

We start with examining the stability of solitary waves for the case $h=2$ by integrating numerically the surface Euler system (2.10-2.13). The fourth-order Runge-Kutta method is used for time integration for $Y$ and $\Phi$, where the time step is chosen as $d t=0.0005$. Due to the periodic assumption and conservation of mass, $\bar{h}(t)$ defined in (2.7) remains unchanged at all time for a particular solution. A depression wave, 

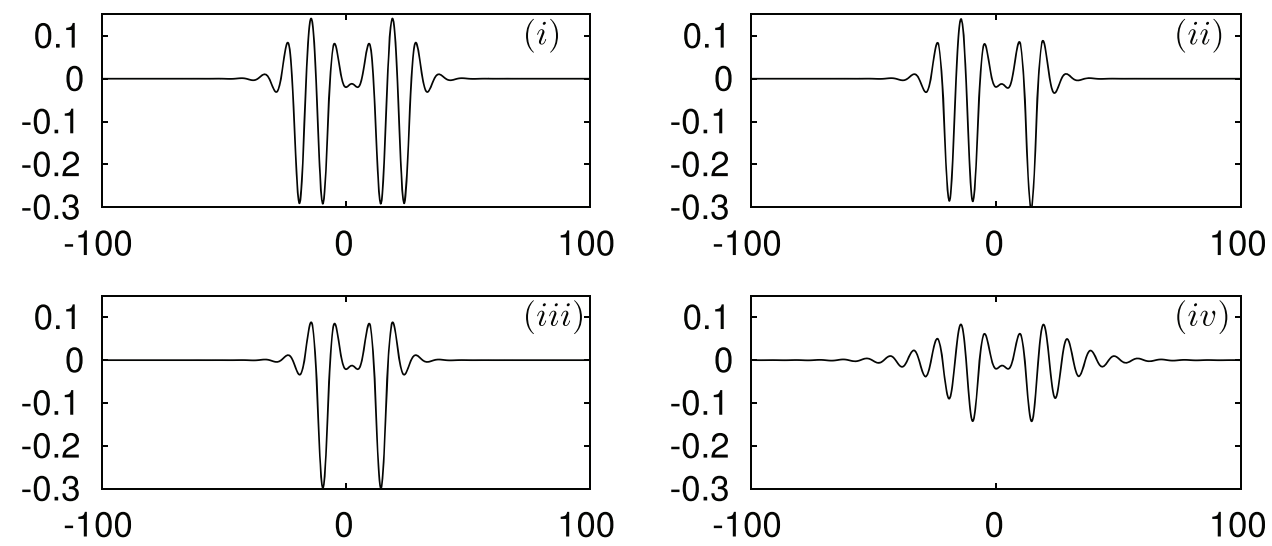

FIG. 7. Typical solitary wave profiles for the points $i-i v$ shown in Fig. 6. All the profiles are plotted in the physical plane.
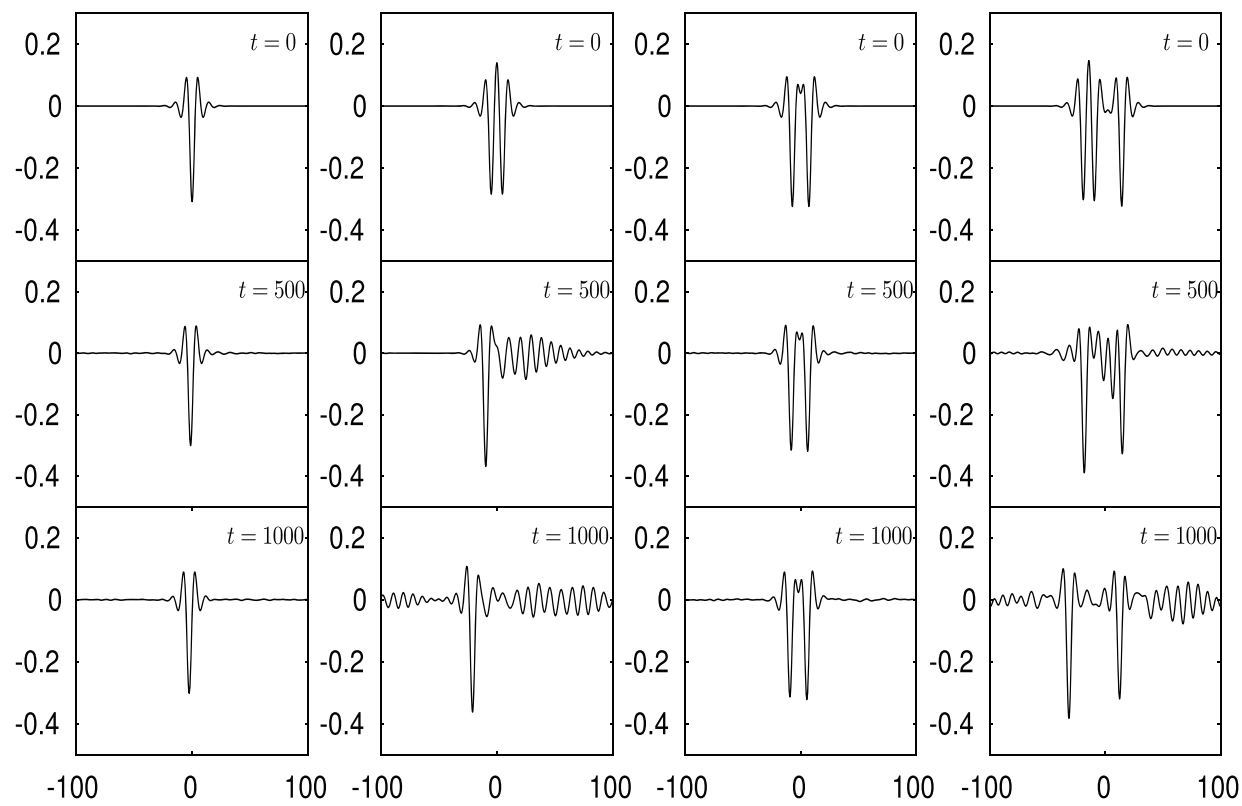

FIG. 8. Time-evolution of a stable depression wave (left), an unstable elevation wave (middle left), a stable elevation wave (middle right) and an unstable asymmetric wave (right) for $h=2$. All the profiles are plotted in the physical plane, and a frame of reference moving at $c=1.2$ is chosen.

two elevation waves and an asymmetric wave travelling with speed $c=1.2$ were considered in the numerical experiments (see Fig. 8). Also a frame of reference moving with the same speed was chosen. An initial perturbation of $+5 \%$ of the amplitude was given to the depression wave whose stability is confirmed since the wave maintains its shape as the computation runs up to $t=1000$ (see the left graph in Fig. 8). It was found earlier from the speed-amplitude bifurcation diagram that the speed decreases as the wave amplitude increases. Hence, the depression wave, which was enlarged by $5 \%$, moves leftwards in the screen with a speed less than that of the reference frame. The second column of pictures from the 


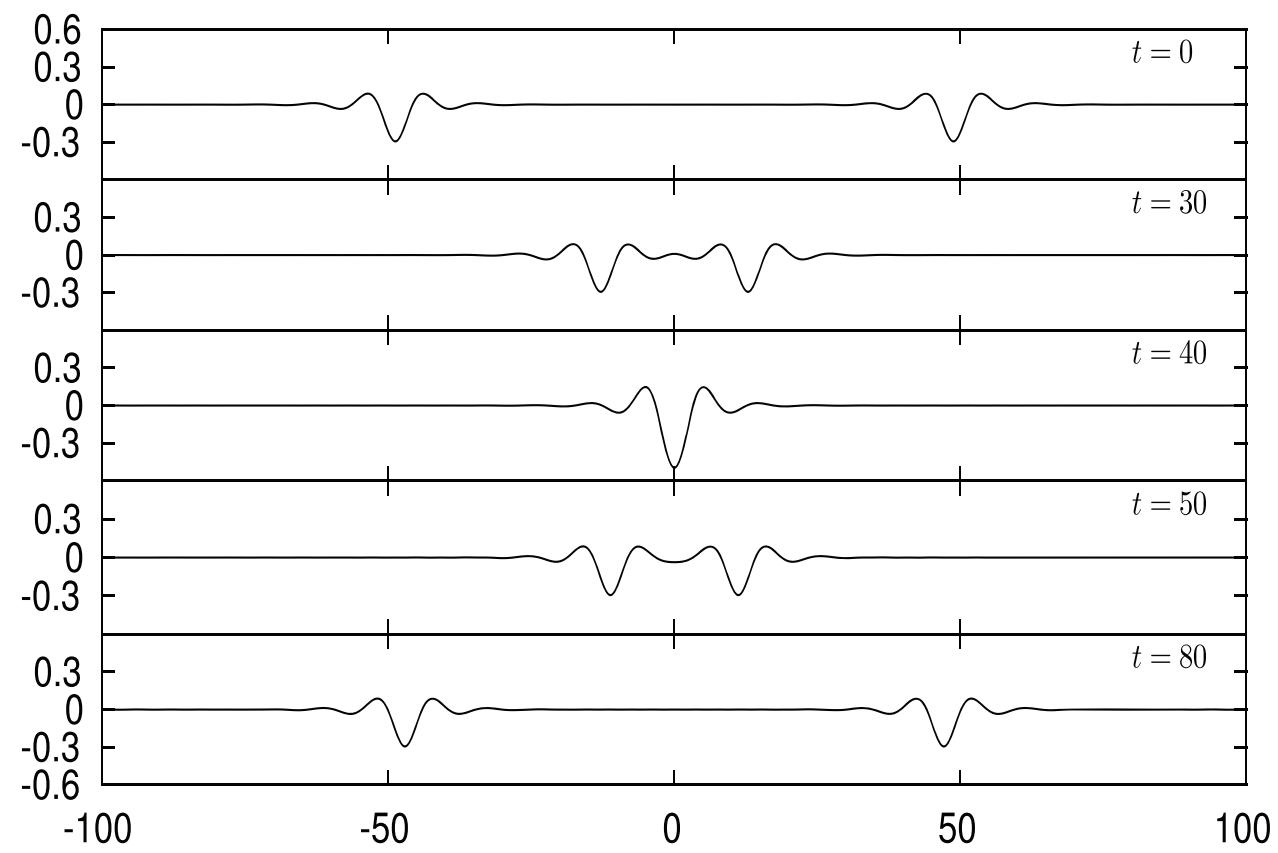

FIG. 9. Head-on collision between two identical depression waves travelling with $c=1.2$. The snapshots are taken at $t=0,30$, 40, 50 and 80 . All the profiles are plotted in the physical plane.

left shows that the elevation solitary wave appears to be unstable. The elevation wave, which initially suffers a $1 \%$ increase in amplitude, eventually evolves into a depression one after a period of time. We performed the same computations with elevation waves of the second kind. It was initially perturbed by a 5\% amplitude increase, and the numerical result confirms the stability of this solution (see the middle right graph of Fig 8). The multi-wavepacket asymmetric solitary wave is also unstable because its left packet, which is in the form of an elevation wave, turns to be a depression wave as presented in the right graph of Fig. 8.

Next numerical experiments on solitary wave interactions are performed for $h=2$ (see Fig. 9). We consider two depression waves placed initially at some distance. They travel with the same speed $c=1.2$ but in the opposite directions. After the collision, both waves survive and continue to travel without losing their main structures. Then we studied head-on collisions between two identical depression waves moving much slower $(c=0.65)$ (see Fig. 10). In this case, the superposition of the waves may cause a problem due to the considerably smaller value of the water depth (recall that $h=2$ ). The waves travel with $c=0.65$ towards each other up to $t=35.5$ when they fully collide. Immediately after that instant, the whole system breaks down because a touch-down singularity takes place.

We finally study the problem of moving loads on an elastic sheet. It can be realized numerically by introducing a pressure distribution moving at a constant speed $U$, e.g.

$$
\mathcal{P}_{e}(x, t)=B \exp \left[-\left(\frac{x+220-U t}{d}\right)^{2}\right],
$$

where $B$ is the amplitude parameter and $d$ measures the width of the distribution. The domain is taken to be $[-250,250]$, and the centre of the pressure distribution is initially placed at $x=-220$. Our first 


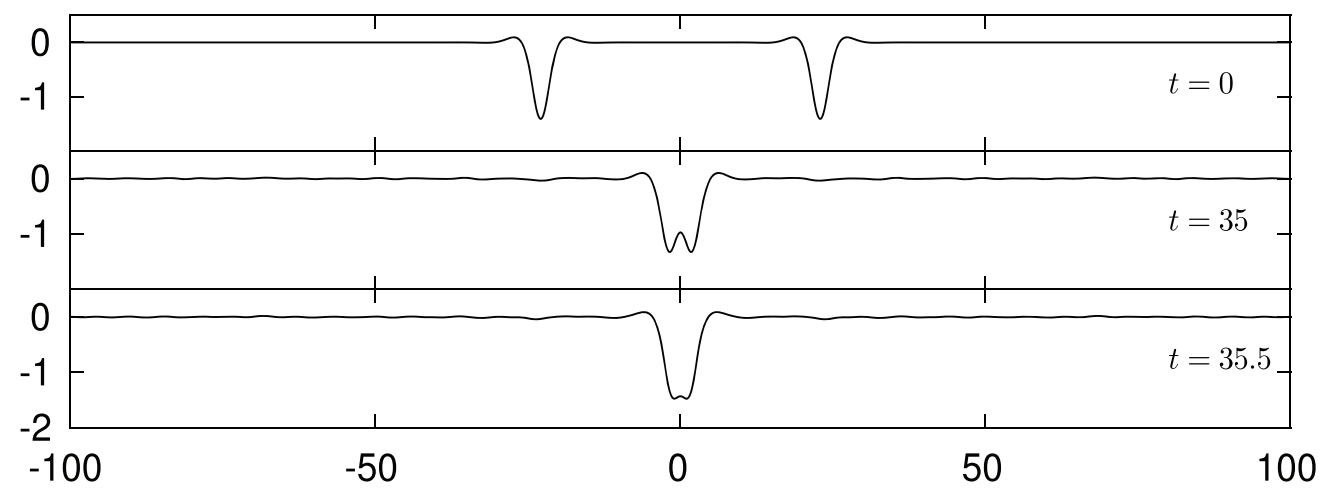

FIG. 10. Head-on collision between two stable depression waves travelling with $c=0.65$. The snapshots are taken at $t=0,35$ and 35.5. All the profiles are plotted in the physical plane. A touch-down singularity takes place after $t=35.5$.

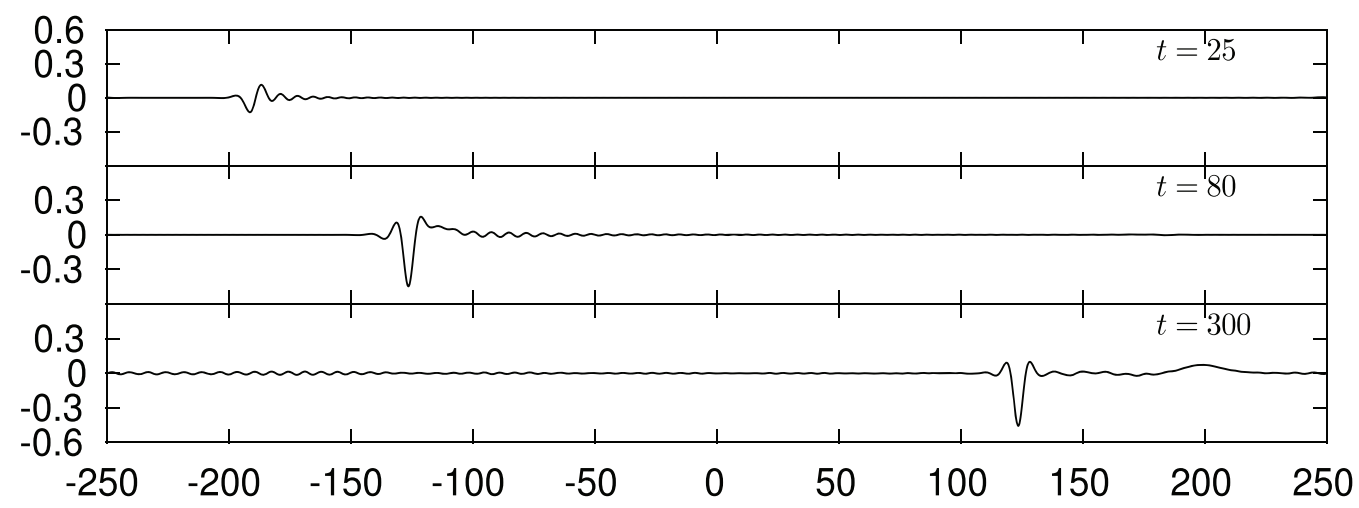

FIG. 11. Numerical experiment of excitation of depression solitary waves. A moving pressure disturbance is initially switched on at $x=-220$, and it is turned off at $t=80$. The snapshots are taken at $t=25,80$ and 300. All the profiles are plotted in the physical plane.

attempt was to excite a stable depression wave by setting $B=0.02, d=1$ and $U=1.2$ which is slightly below the phase speed minimum. The moving disturbance was switched on at $t=0$ and off at $t=80$, and the computation is continued up to $t=300$. As shown in Fig. 11, a stable depression wave was generated and can coexist with the background noise for long time. Next we attempt to excite a stable elevation wave by applying a disturbance which is the sum of two identical Gaussian pressures separated by a distance $l$, namely,

$$
\mathcal{P}_{e}(x, t)=B \exp \left[-\left(\frac{x+220-U t}{d}\right)^{2}\right]+B \exp \left[-\left(\frac{x+220-l-U t}{d}\right)^{2}\right] .
$$

We choose $B=0.05, l=10, d=1$ and $U=1$.2. The disturbance is switched on at $t=0$ and off at $t=100$. From the results shown in Fig. 12, the generation of a stable elevation wave is also achieved.

In the last numerical experiment, we use two large Gaussian distributions with the following set up: $B=0.1, d=1, l=10$ and $U=1.2$. They were initially switched on at $x=-250$ and turned off at $t=125$. A larger domain $L=300$ was chosen. After the removal of the pressure, two stable depression 


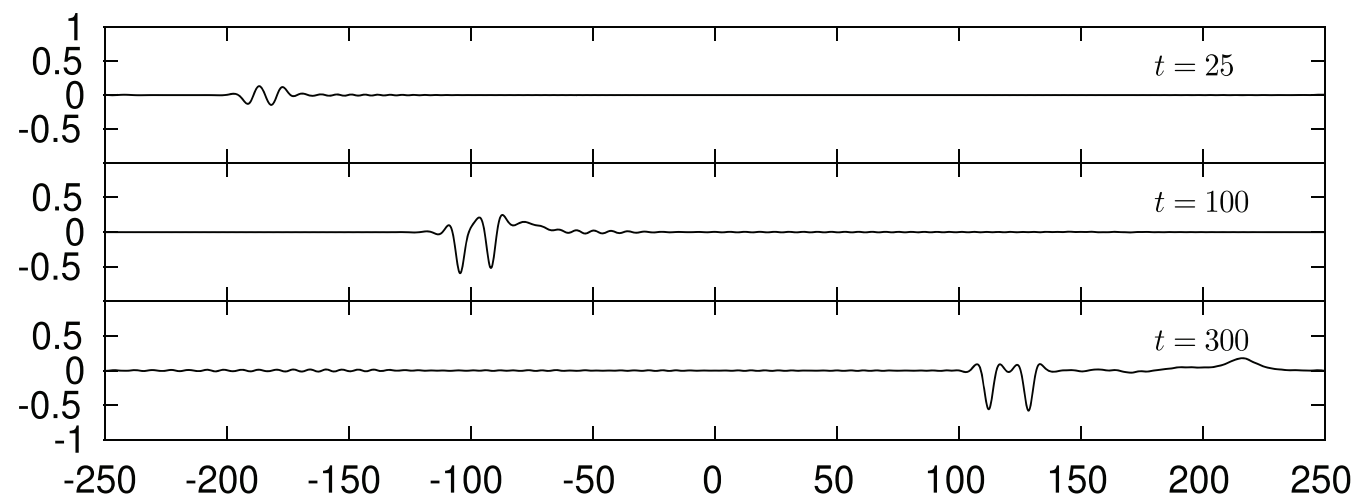

FIG. 12. Numerical experiment of excitation of stable elevation solitary waves. A moving disturbance is switched on at $t=0$ and off at $t=100$. The snapshots are taken at $t=25,100$ and 300. All the profiles are plotted in the physical plane.

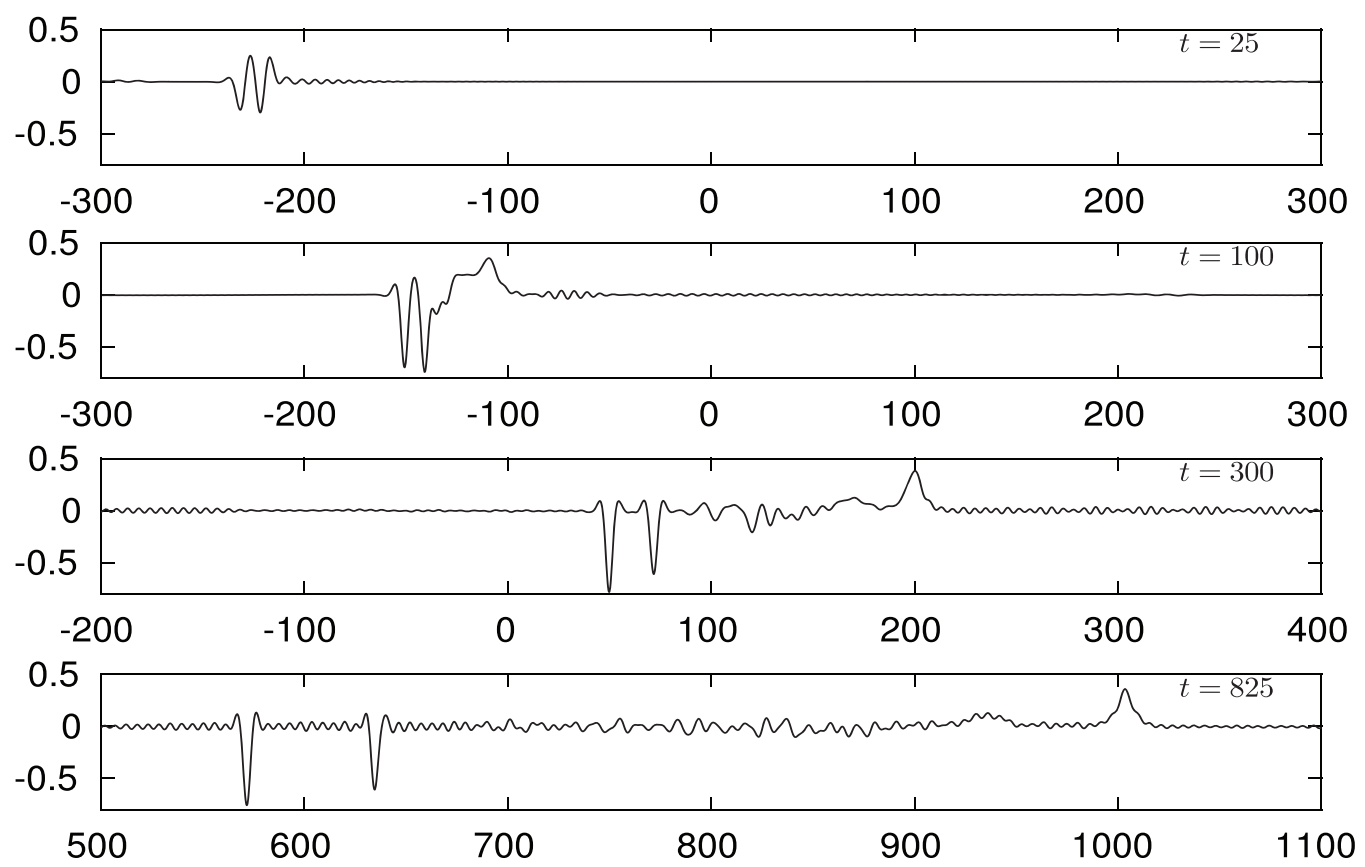

FIG. 13. Numerical experiment of excitation of generalized solitary wave. A moving disturbance is switched on at $t=0$ and off at $t=125$. The snapshots are taken at $t=25,125,300$ and 825 . All the profiles are plotted in the physical plane.

waves of different amplitudes were generated. More interestingly, a single pulse with oscillating ripples on both sides, which is the so-called generalized solitary wave, has been observed up front. Its generation is due to the conservation of mass of water in the finite strip. The generalized solitary wave keeps propagating rightwards, with a speed about $1.5\left(>c_{0}=\sqrt{2}\right)$ as shown by the numerical observation, up to $t=825$ (see Fig. 13). We compare the excited generalized solitary wave with the steady solutions computed by series truncation method in the study by Gao \& Vanden-Broeck (2014). With $c \simeq 1.5$ and $h=2$, the corresponding parameters $F$ and $\beta$ in that paper can be estimated by noting that in terms of 


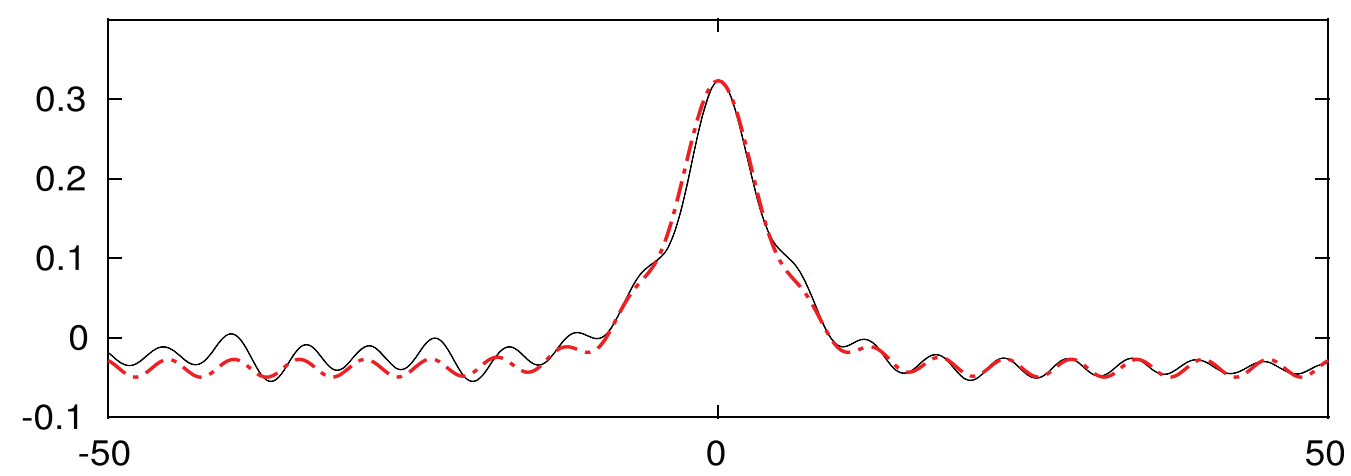

FIG. 14. Comparison of the excited generalized solitary wave (solid curve) as shown in Fig. 13 and the steady solution from Gao $\&$ Vanden-Broeck (2014) (dashed-dotted curve) where the corresponding parameters are $F=1.063$ and $\beta=0.055$. The profiles are plotted in the physical plane.

our dimensionless variables $F=c h^{-1 / 2}, \beta=c^{-2} h^{-3}$. This gives $F=1.063$ and $\beta=0.055$. As shown in Fig. 14, the fully nonlinear steady solution from the study by Gao \& Vanden-Broeck (2014), with $F=1.063$ and $\beta=0.055$, agrees well with the excited generalized solitary wave. To our knowledge, this is the first time that generalized solitary wave is excited numerically by moving loads with a speed in the transcritical regime. It is worth noting that Guyenne \& Părău (2014) also observed generalized solitary waves in the regime of $U>c_{0}$ via direct numerical simulations of a truncated system, and their results only showed a dispersive tail on one side of the central pulse.

\section{Conclusion}

We investigated the problem of flexural-gravity waves in water of arbitrary depth, and particular attention was paid to the influence of finite-depth effects. The bifurcations of solitary waves were studied for $h=300$ (deep water) and $h=2$. Fully nonlinear time-dependent computations were carried out to examine the stability characteristics of different types of solitary waves. Numerical experiments of head-on collision between identical depression waves were carried out, and a touch-down singularity was observed when the wave amplitude is considerably larger. The problem of moving loads on the elastic sheet was also considered. Single and multiple moving pressure distributions were used to excite depression solitary waves and stable elevation ones. Generalized flexural-gravity solitary waves, whose existence was previously predicted by ideal steady computations, were also excited by time-dependent simulations in the present paper.

\section{Funding}

EPSRC (EP/N018559/1 to J.M.V.B.); National Natural Science Foundation of China (11772341 to Z.W.); Key Research Program of Frontier Sciences, CAS (QYZDB-SSW-SYS015 to Z.W.); Strategic Priority Research Program of the Chinese Academy of Sciences (XDB22040203 to Z.W.).

\section{REFERENCES}

Alam, M.-R. (2013) Dromions of flexural-gravity waves. J. Fluid Mech., 719, 1-13.

Ashton, G. D. (1986) River and Lake Ice Engineering. Littleton, CO: Water Resources Publication.

Choi, W. \& Camassa, R. (1999) Exact evolution equations for surface waves. J. Eng. Mech., 125, 756-760. 
Dyachenko, A. I., Zakharov, V. E. \& Kuznetsov, E. A. (1996) Nonlinear dynamics of the free surface of an ideal fluid. Plasma Phys. Rep., 22, 829-840.

Forbes, L. K. (1986) Surface waves of large amplitude beneath an elastic sheet. Part 1. High-order series solution. J. Fluid Mech., 169, 409-428.

Forbes, L. K. (1988) Surface waves of large amplitude beneath an elastic sheet. Part 2. Galerkin solution. J. Fluid Mech., 188, 491-508.

Gao, T. \& Vanden-Broeck, J.-M. (2014) Numerical studies of two-dimensional hydroelastic periodic and generalised solitary waves. Phys. Fluids, 26, 087101.

GaO, T., WANG, Z. \& VANDEN-BroecK, J.-M. (2016) New hydroelastic solitary waves in deep water and their dynamics. J. Fluid Mech., 788, 469-491.

GuYENnE, P. \& PĂRĂU, E. I. (2012) Computations of fully nonlinear hydroelastic solitary waves on deep water. J. Fluid Mech., 713, 307-329.

GuYenne, P. \& PĂRĂU, E. I. (2014) Finite-depth effects on solitary waves in a floating ice sheet. J. Fluids Struct., 49, 242-262.

Korobkin, A., PĂRĂU, E. I. \& VANDEn-Broeck, J.-M. (2011) The mathematical challenges and modelling of hydroelasticity. Philos. Trans. R. Soc. A, 369, 2803-2812.

Li, Y. A., Hyman, J. M. \& ChOI, W. (2004) A numerical study of the exact evolution equations for surface waves in water of finite depth. Stud. Appl. Math., 113, 303-324.

Marko, J. R. (2003) Observations and analyses of an intense waves-in-ice event in the Sea of Okhotsk. J. Geophys. Res., 108, 3296.

Milewski, P. \& Wang, Z. (2013) Three dimensional flexural-gravity waves. Stud. Appl. Math., 131, 135-148.

Milewski, P. A., VAnden-Broeck, J.-M. \& WANG, Z. (2010) Dynamics of steep two-dimensional gravity-capillary solitary waves. J. Fluid Mech., 664, 466-477.

Milewski, P. A., Vanden-Broeck, J.-M. \& Wang, Z. (2011) Hydroelastic solitary waves in deep water. J. Fluid Mech., 679, 628-640.

PĂRĂU, E. I. \& DiAs, F. (2002) Nonlinear effects in the response of a floating ice plate to a moving load. J. Fluid Mech., 460, 281-305.

PĂRĂU, E. I. \& VANDEN-Broeck, J.-M. (2011) Three-dimensional waves beneath an ice sheet due to a steadily moving pressure. Philos. Trans. R. Soc. A, 369, 2973-2988.

Plotnikov, P. \& Toland, J. F. (2011) Modelling nonlinear hydroelastic waves. Philos. Trans. R. Soc. A, 369, 2942-2956.

Squire, V., Hosking, R. J., Kerr, A. D. \& Langhorne, P. J. (1996) Moving Loads on Ice Plates. Solid Mech. Appl., vol. 45. Kluwer.

Squire, V., Robinson, W., Langhorne, P. \& Haskell, T. (1988) Vehicles and aircraft on floating ice. Nature, 333, 159-161.

TAKIZAWa, T. (1985) Deflection of a floating sea ice sheet induced by a moving load. Cold Regions Sci. Tech., 11, $171-180$.

Takizawa, T. (1987) Field studies on response of a floating sea ice sheet to a steadily moving load. Contrib. Inst. Low Temp. Sci. A, 36, 31-76.

TAKizawa, T. (1988) Response of a floating sea ice sheet to a steadily moving load. J. Geophys. Res., 93, $5100-5112$.

VAnden-Broeck, J.-M. \& PăRĂU, E. I. (2011) Two-dimensional generalized solitary waves and periodic waves under an ice sheet. Philos. Trans. R. Soc. A, 369, 2957-2972.

WAng, Z., \& Milewski, P. A. (2012) Dynamics of gravity-capillary solitary waves in deep water. J. Fluid Mech., 708, 480-501.

Wang, Z., Milewski, P. \& Vanden-Broeck, J.-M. (2014) Computation of three-dimensional flexural-gravity solitary waves in arbitrary depth. Proc. IUTAM, 11, 119-129.

Wang, Z., Vanden-Broeck, J.-M. \& Milewski, P. (2014) Asymmetric gravity-capillary solitary waves on deep water. J. Fluid Mech., 759. 